\title{
Fractional Brownian motion of director fluctuations in nematic ordering
}

\author{
Zhang, Z.; Mouritsen, Ole G.; Otnes, K.; Riste, T.; Zuckermann, Martin J.
}

Published in:

Physical Review Letters

Link to article, DOI:

10.1103/PhysRevLett.70.1834

Publication date:

1993

Document Version

Publisher's PDF, also known as Version of record

Link back to DTU Orbit

Citation $(A P A)$ :

Zhang, Z., Mouritsen, O. G., Otnes, K., Riste, T., \& Zuckermann, M. J. (1993). Fractional Brownian motion of director fluctuations in nematic ordering. Physical Review Letters, 70(12), 1834-1837.

https://doi.org/10.1103/PhysRevLett.70.1834

\section{General rights}

Copyright and moral rights for the publications made accessible in the public portal are retained by the authors and/or other copyright owners and it is a condition of accessing publications that users recognise and abide by the legal requirements associated with these rights.

- Users may download and print one copy of any publication from the public portal for the purpose of private study or research.

- You may not further distribute the material or use it for any profit-making activity or commercial gain

- You may freely distribute the URL identifying the publication in the public portal 


\title{
Fractional Brownian Motion of Director Fluctuations in Nematic Ordering
}

\author{
Z. Zhang, ${ }^{(1)}$ O. G. Mouritsen, ${ }^{(2)}$ K. Otnes, ${ }^{(3)}$ T. Riste, ${ }^{(3)}$ and M. J. Zuckermann ${ }^{(1)}$ \\ ${ }^{(1)}$ Centre for the Physics of Materials, Department of Physics, McGill University, Montréal, Québec, Canada H3A $2 T 8$ \\ ${ }^{(2)}$ Canadian Institute of Advanced Research and Department of Physical Chemistry, The Technical University of Denmark, \\ Building 206, DK-2800 Lyngby, Denmark \\ ${ }^{(3)}$ Institutt for Energeteknikk, POB 40, N-2007, Kjeller, Norway
}

(Received 11 November 1992)

\begin{abstract}
Temporal director fluctuations in nematic ordering were studied by computer simulation on the Lebwohl-Lasher model as well as by neutron-scattering experiments on the nematogen d-PAA. The time-series data have been analyzed by the rescaled-range method and in terms of the power spectrum in order to determine the Hurst exponent $H$. Theory and experiment are in good agreement. A value of $H \simeq 1$ was found for the nematic phase, characterizing fractional Brownian motion, whereas $H \simeq 0.5$, reflecting ordinary Brownian motion, applies in the isotropic phase. Field-induced crossover from fractional to ordinary Brownian motion was observed in the nematic phase.

PACS numbers: $64.70 . \mathrm{Md}, 05.40 .+\mathrm{j}, 64.60 . \mathrm{Cn}$
\end{abstract}

The study of temporal fluctuations in complex dynamical systems has been revived through the concept of fractional Brownian motion introduced by Mandelbrot [1]. In contrast to ordinary Brownian motion which reflects independent stochastic processes, fractional Brownian motion implies persistence or antipersistence and power-law correlations in the fluctuations. An interesting possibility for observing fractional Brownian motion in a cooperative many-particle system exists in the case of fluctuating modes in a symmetry-broken state of continuous degeneracy. A candidate for a system exhibiting this type of behavior is a liquid crystal within the nematic phase where the director fluctuations correspond to a dynamical mode which is critical for all temperatures in the nematic phase [2].

In this Letter we present results from a combined theoretical and experimental study of the director fluctuations in nematic ordering. The theoretical calculations are based on computer simulations on the most basic statistical mechanical model, the three-dimensional Lebwohl-Lasher model [3], of the nematic-isotropic orientational phase transition in liquid crystals. The experimental study is based on neutron-scattering measurements on the nematogenic substance deuterated paraazoxy-anisole (d-PAA). Both the theoretical and experimental study lead to equilibrium time series of the nematic director. From statistical analysis of these time series in terms of the autocorrelation function $C(t)$ and the power spectrum $P(f)$ of the accumulated fluctuations we obtained the following results: (i) the correlation of the accumulated director fluctuations scales as $C(t) \sim t^{2 H}$ and the power spectrum as $P(f) \sim f^{-(2 H+1)}$ with the value of the Hurst exponent being $H \simeq 1$ for all temperatures in the nematic phase; (ii) in the isotropic phase, $C(t)$ scales as $C(t) \sim t^{2 H}$ and $P(f)$ scales as $P(f) \sim f^{-(2 H+1)}$ with $H \simeq 0.5$ independent of temperature; and (iii) in the presence of a unidirectional ordering field there is a crossover from $H \simeq 1$ to $H \simeq 0.5$ in the nematic phase, whereas the pres- ence of the field does not affect the Hurst exponent in the isotropic phase. For comparison we show that the correlation function of the order-parameter fluctuations and the corresponding power spectrum is characterized by a Hurst exponent, $H \simeq 0.5$, independent of the phase, independent of the temperature, and independent of the presence of an ordering field. These results show that the director fluctuations exhibit fractional Brownian motion, $H>\frac{1}{2}[1,4,5]$ in the nematic phase, whereas whenever the continuous degeneracy is broken, the fluctuations follow ordinary Brownian motion, $H=$ $\frac{1}{2}$. A similar crossover behavior with the same Hurst exponent values has also been observed for the fluctuations in the magnetization direction in the isotropic threedimensional Heisenberg ferromagnet [6]. The agreement between experimental data for a specific nematogenic substance and results from calculations on a simple parameter-free model, as reported in the present paper, therefore suggests that fractional Brownian motion characterized by a Hurst exponent value $H=1$ may be generic for director fluctuations of continuous symmetry.

The Lebwohl-Lasher model [3] is the lattice version of the classic Maier-Saupe model [7] of an anisotropic liquid in which the molecules are coupled by the Hamiltonian

$$
\mathcal{H}=-\epsilon \sum_{i, j} P_{2}\left(\cos \theta_{i j}\right),
$$

where $P_{2}\left(\cos \theta_{i j}\right)=\frac{1}{2}\left(3 \cos ^{2} \theta_{i j}-1\right), \theta_{i j}$ is the angle between the axes of rotor molecules at nearest-neighbor sites $i$ and $j$, and $\epsilon$ is a coupling parameter. The LebwohlLasher model, which neglects the translational variables and the steric effects of the rotor molecules, is known to produce a very weak first-order orientational phase transition $[8,9]$. The nematic order in the Lebwohl-Lasher model is characterized by a second-rank tensor order parameter, Q. As a result of the continuous degeneracy of the nematic ordering in the absence of a field, the direction of the nematic director varies and has to be determined during the simulation in order to calculate the 
value of the orientational order parameter, $\left\langle P_{2}\left(\cos \theta_{i}\right)\right\rangle$, where $\theta_{i}$ is the angle between the axis of the $i$ th rotor and the nematic director. This calculation is facilitated by diagonalization of $\mathbf{Q}, Q_{\alpha \beta}=\sum_{i}\left(\frac{3}{2} u_{\alpha, i} u_{\beta, i}-\frac{1}{2} \delta_{\alpha \beta}\right)$, $\alpha, \beta=x, y, z$, where $u_{\alpha, i}$ is the $\alpha$ component of a unit vector, $\mathbf{u}_{i}$, which specifies the orientation of the $i$ th molecule. The instantaneous value of the order parameter is then given by the largest eigenvalue.

Using Monte Carlo computer-simulation techniques, we have calculated equilibrium time series of the nematic director, given by the components $u_{\alpha}(t)$ of the unit directional vector, $\mathbf{u}=\sum_{i} \mathbf{u}_{i}$, as well as the nematic order parameter. The calculations are carried out on a simple cubic lattice with periodic boundary conditions and $L^{3}=28^{3}$ lattice sites. The simulations are performed using Glauber dynamics involving attempts to randomly rotate the direction of the individual molecules. Time is measured in units of Monte Carlo steps per lattice site. The Glauber dynamics, which correspond to the overdamped regime, does not conserve the nematic order. However, except in the transition region where the order parameter exhibits strong fluctuations, we expect our stochastic model dynamics to resemble that of the true dynamics. The time series have been determined for different temperatures in both the nematic and the isotropic phase in the absence as well as in presence of an ordering-field term, $-h_{\theta}^{2} \sum_{i} \cos ^{2} \theta_{i}$, added to the Hamiltonian in Eq. (1).

The neutron-scattering experiments on director fluctuations are carried out on samples of d-PAA. The study is an extension of earlier work [10] where it was found that the Hurst exponent has the value $H \simeq 0.5$ in the solid phase, in the isotropic liquid phase, and in the magnetically aligned nematic phase, whereas at zero magnetic field in the nematic phase the Hurst exponent assumed the value $H \simeq 0.74$. This latter value was suspected to be influenced by effects due to convection flow alignment which tends to reduce the effective value of $H$. In the present Letter we report new experimental results for the case where the convective state has been carefully avoided [11].

The sample volume irradiated by the incident neutron beam was $(0.3 \times 0.3 \times 1) \mathrm{cm}^{3}$ with the long dimension along the scattering vector. Intensity versus time series were recorded for the liquid diffraction peak at $q=1.8$ $\AA^{-1}$. It is essential that, for a given temperature, the intensity of this peak, apart from material constants, depends only on the orientation of the director relative to the scattering vector [12]. Orientational fluctuations, therefore, manifest themselves as temporal variation of the intensity. Convective flow may change the average orientation of the director and reduce the level of fluctuations. By carefully measuring the time-averaged neutron intensity as a function of the applied vertical temperature gradient, for both positive and negative gradients, we made sure that the effect of convective flow has been minimized as much as possible in the present work. Strong anchoring of the director at the container walls may reduce the fluctuations, in the same way as the magnetic field. For a sample container of the present size, the influence of the anchoring field is expected to be small. This expectation was confirmed by a series of measurements of the time-averaged neutron intensity as a function of the applied field. The sensitivity of the intensity, and hence also of the director fluctuations, to fields in the range of 10 Oe shows that the anchoring field is too weak to suppress the thermal fluctuations in the bulk of the sample. A time series was typically $11 \mathrm{~h}$ long, consisting of 2000 channels of $20 \mathrm{~s}$ time width. With this arrangement one can directly record collective orientational fluctuations of spatial and temporal correlation lengths of the order of $\gtrsim 0.3 \mathrm{~cm}$ and $\gtrsim 20 \mathrm{~s}$, respectively. This implies that only director fluctuations are detected, and not order-parameter fluctuations, except perhaps close to the nematic-isotropic transition.

The time series obtained from both the computer simulations and the neutron-scattering experiments have been analyzed by the rescaled-range $(R / S)$ method $[1,5]$ which was originally developed by Hurst [13] to analyze statistical fluctuations of water levels in large natural reservoirs. In the case of a time series, $u(t)$, the $R / S$ analysis is based on a range $R=\max [X(t, \tau)]-\min [X(t, \tau)], 0 \leq t \leq \tau$, and a standard deviation $S=\left(\frac{1}{\tau} \sum_{t=1}^{\tau}\left[u(t)-\langle u\rangle_{\tau}\right]^{2}\right)^{1 / 2}$, where the average of the stochastic variable $u(t)$ over the time range $\tau$ is $\langle u\rangle_{\tau}=\tau^{-1} \sum_{t=1}^{\tau} u(t)$ and the accumulated fluctuations over the same time range are $X(t, \tau)=\sum_{t^{\prime}=1}^{t}\left[u\left(t^{\prime}\right)-\langle u\rangle_{\tau}\right]$. Based upon these variables, an autocorrelation function $C(t)$ can be defined as $C(t)=\langle X(0) X(t)\rangle$. Scaling now implies [5],

$$
R / S \sim \tau^{H}, \quad C(t) \sim t^{2 H},
$$

where $H$ is the Hurst exponent. The power spectrum is expected to scale as [5]

$$
P(f)=\left|\int X(t) \exp (-i 2 \pi f t) \mathrm{d} t\right|^{2} \sim f^{-\beta}
$$

with $\beta=2 H+1$. Statistical independence implies $H=\frac{1}{2}$ and ordinary Brownian motion. In the case of $H \neq \frac{1}{2}$, the correlation function has power-law decay and infinitely long correlations. This latter case is associated with fractional Brownian motion [1].

In the case of the model simulations, $u(t)$ is taken to be the calculated time series for the director described by one of its Cartesian components, $u_{\alpha}(t)$. All three components are found to behave statistically similarly. A selection of the data for the $R / S$ values, as a function of time range $\tau$, obtained for the director fluctuations in the nematic phase for different values of the symmetrybreaking field, $h_{\theta}$, is given in Fig. 1. For zero field the data show a clear scaling behavior, Eq. (2), over the full time range with a Hurst exponent value $H \simeq 1$. As the field value is increased, a crossover is found to a different 


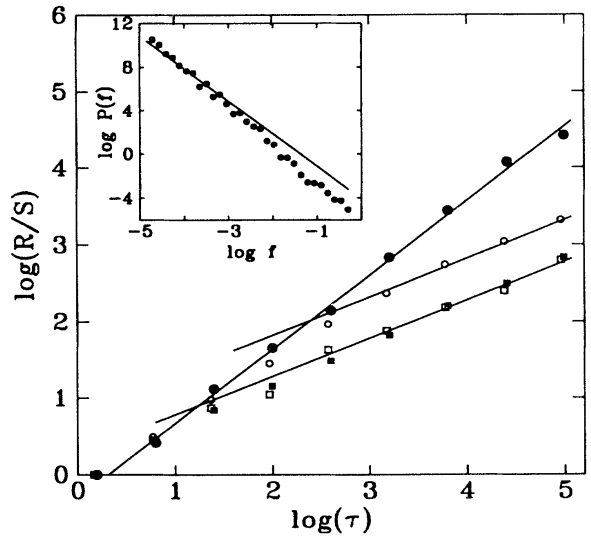

FIG. 1. Log-log plot of the $R / S$ value vs time range $\tau$ for the nematic director fluctuations obtained from computer simulation of the Lebwohl-Lasher model at a temperature in the nematic phase, $T=1.00 \epsilon / k_{B}$. The data correspond to four different values of the applied field, $h_{\theta}^{2}=$ $0(\bullet), 0.05(0), 0.4(\square)$, and $1(\square)$. The best linear fits to the data sets over long time ranges are given by the solid lines, $R / S \sim \tau^{H}$, with $H \simeq 1$ for zero field and $H \simeq 0.5$ for finite fields. The inset shows the power spectrum $P(f)$ for the zero-field data.

scaling behavior characterized by $H \simeq 0.5$. The crossover occurs at shorter time ranges, the stronger the value of the field. In the crossover regime, effective-exponent values between 0.5 and 1 can be assigned to the data. These results are independent of temperature in the nematic phase. In the isotropic phase the data for the finitesystem director fluctuations demonstrate that ordinary Brownian motion applies over long time ranges independent of temperature and independent of the strength of an applied field. In contrast to the fluctuations in the nematic director (in the absence of a field), the fluctuations in the nematic order parameter correspond to independent stochastic processes and hence ordinary Brownian motion $\left(H=\frac{1}{2}\right)$ in either phase and are independent of temperature and the application of a field, as illustrated by the data in Fig. 2.

We now turn to the experimental data in which the stochastic variable $u(t)$ is the scattering intensity from the director fluctuations [10]. The data are analyzed by the same methods as the numerical data. Figure 3 shows data from the $R / S$ analysis for different values of the magnetic field applied normal to the scattering vector. We observe the same general behavior as in Fig. 1. At zero field, the data are described by a Hurst exponent, $H \simeq 1$, over the full time range whereas for increasing field values there is an effective crossover to lower values of $H$ and ultimatively at high fields, $H \simeq 0.5$. It is not possible to resolve a crossover region in the experimental data. The variation of the effective experimental value of $H$ is shown in Fig. 4. The field dependence of the scattering intensity of the liquid diffraction peak, shown

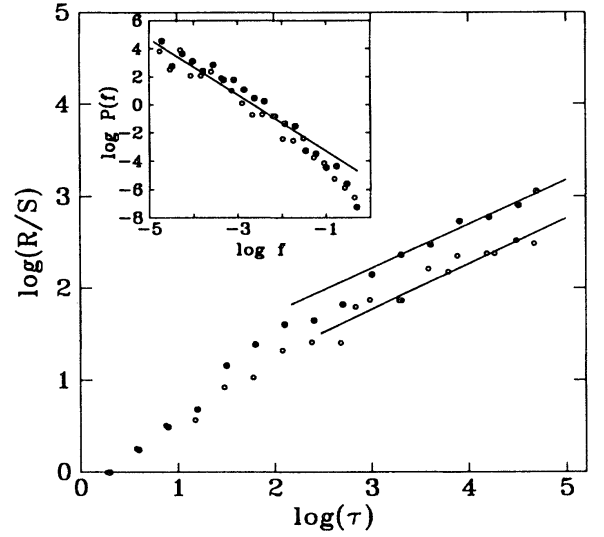

FIG. 2. Log-log plot of the $R / S$ value vs time range $\tau$ for the nematic order-parameter fluctuations obtained from computer simulation of the Lebwohl-Lasher model for a temperature, $T=1.00 \epsilon / k_{B}(\bullet)$, in the nematic phase and a temperature, $T=1.30 \epsilon / k_{B}(\circ)$, in the isotropic phase. The best linear fit to the entire data set over long time ranges is given by the solid lines, $R / S \sim \tau^{H}$, with $H \simeq 0.5$. The inset shows the corresponding data for the power spectra $P(f)$.

in the inset in Fig. 4, reflects the gradual approach to a unique director for the whole sample.

Some results for the power spectrum $P(f)$ of the director fluctuations at zero field are shown as insets in Figs. 1, 2, and 3 for the theoretical and experimental data, respectively. It is seen that, although the statistical analysis of the power spectra is more uncertain, $P(f)$ also obeys scaling over a considerable frequency range

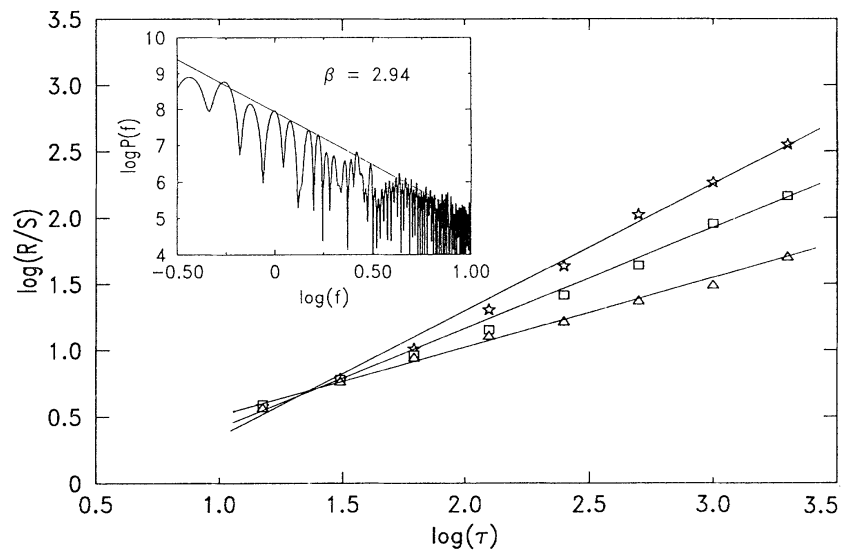

FIG. 3. Log-log plot of the $R / S$ value vs time range $\tau$ for the nematic director fluctuations obtained from neutron scattering measurements on d-PAA in the nematic phase $(T=$ $398 \mathrm{~K}$ ) for different values of the magnetic field $h$. Data are shown for $h=0(\star), 54(\square)$, and $100(\triangle)$ Oe. The solid lines indicate fits of the scaling form, $R / S \sim \tau^{H}$, to the data with resulting values of the Hurst exponent shown in Fig. 4. The inset shows the data for the power spectrum $P(f)$ (in the case of $h=0$ ) and a solid line corresponding to $\beta=2 H+1$. 


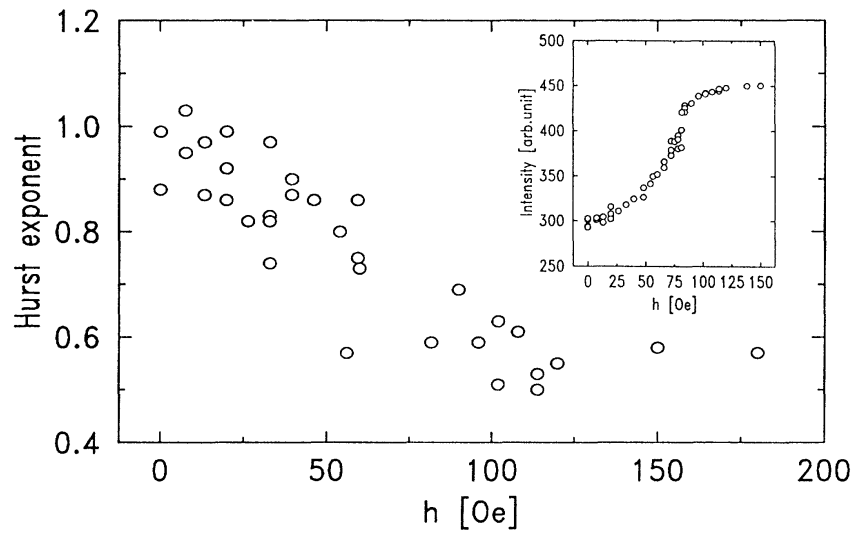

FIG. 4. Plot of the effective Hurst exponent obtained from the $R / S$ analysis of the nematic director-fluctuation data obtained from neutron-scattering measurements on d-PAA. The inset shows the scattering intensity of the liquid diffraction peak as a function of magnetic field.

and that the scaling relation, $\beta=2 H+1$, is approximately fulfilled.

The physical interpretation of the results from the present combined theoretical and experimental investigation of director fluctuations in liquid crystals in addition to those reported earlier [10] is as follows: In the ordered nematic phase, the director is subject to a continuous degeneracy since its direction is not coupled to the lattice and there is no activation barrier for directional rotation. Hence the director field is subject to critical fluctuations and power-law correlations for all temperatures within the nematic phase [2]. This leads to fractional Brownian motion of the director. In contrast, the nematic order parameter is not a critical mode (except at $T_{\mathrm{c}}[9]$ ), and the order-parameter fluctuations in both phases are of short range and are associated with ordinary Brownian motion. The continuous degeneracy of the nematic director can be lifted by an ordering field, in which case the fluctuations become quenched and the mode is no longer critical. Ordinary Brownian motion then results over long time ranges, as observed both in the experiments and in the simulations. At short time ranges, however, the symmetry-breaking field is not capable of destroying the power-law correlations and only at longer ranges is there a crossover to ordinary Brownian motion. This crossover occurs for shorter time ranges, as the field increases.

We wish to thank Hans C. Fogedby and Jens Feder for stimulating comments and suggestions. The work was supported by FCAR du Quebec under a centre and team grant, by NSERC of Canada, and by the Danish Natural Science Research Council under Grant No. 117785. The Danish Research Academy supported M.J.Z. and Z.Z. during their stay in Denmark under Grant No. S910015.

[1] B. Mandelbrot, The Fractal Geometry of Nature (Freeman, San Francisco, 1982), p. 247.

[2] P. G. de Gennes, The Physics of Liquid Crystals (Clarendon, Oxford, 1974).

[3] P. A. Lebwohl and G. Lasher, Phys. Rev. A 6, 426 (1972); 7, 2222(E) (1973).

[4] J. Feder, Fractals (Plenum, New York, 1988).

[5] J. Feder, in Spontaneous Formation of Space-Time Patterns and Criticality, edited by T. Riste and D. Sherrington (Kluwer, Dordrecht, 1991), p. 113.

[6] Z. Zhang, O. G. Mouritsen, and M. J. Zuckermann (unpublished).

[7] W. Maier and A. Saupe, Z. Naturforsch. 14A, 882 (1959); 15A, 287 (1960).

[8] U. Fabbri and C. Zannoni, Mol. Phys. 58, 763 (1986).

[9] Z. Zhang, O. G. Mouritsen, and M. J. Zuckermann, Phys. Rev. Lett. 69, 2803 (1992).

[10] K. Otnes and T. Riste, Phys. Scr. T44, 77 (1992).

[11] A full report of the experimental work, including measurements of the convection threshold, the Fredericksz field, and the dependence of the Hurst exponent on the applied temperature gradient, will be published elsewhere [T. Riste and K. Otnes (to be published)].

[12] M. Kohli, K. Otnes, R. Pynn, and T. Riste, Z. Phys. B 24, 147 (1976).

[13] H. E. Hurst, Trans. Am. Soc. Civ. Eng. 116, 770 (1951). 